Lepr Rev (1994) 65, 253-261

\title{
The predictive value of sensation testing in the development of neuropathic ulceration on the hands of leprosy patients
}

\author{
M. KUIPERS* $\ddagger \&$ T. SCHREUDERS $\dagger$ \\ *University of Utrecht Medical School; and $\nmid M c K e a n$ Rehabilita- \\ tion Centre, PO Box 53, Chiang Mai 50000, Thailand
}

Accepted for publication 28 February 1994

Summary The early detection of the loss of protective sensation in leprosy patients is vital if neuropathic ulceration and subsequent disabilities are to be avoided. The aim of this study was to find protective value of sensory thresholds in the hands of leprosy patients.

Thresholds for touch-pressure, vibration and temperature were assessed in areas on leprosy-affected hands near ulcers or ulcer scars (LU-group), in areas without lesions (LN-group), and in controls (N-group). Semmes-Weinstein monofilaments were used for testing the touch-pressure threshold (PST), a biothesiometer for the vibration threshold (VST) and a Thermo Sensation Tester for the temperature threshold (TST).

The distribution of ulcers was about equal on palmar and dorsal aspects of the hands. In the LU-group there was a negative response to SWF of $2.0 \mathrm{~g}$ in all patients, while $74 \%$ could feel the $2.0 \mathrm{~g}$ in $\mathrm{LN}$-areas and in $\mathrm{N}$-areas $100 \%$ could detect the $2.0 \mathrm{~g} \mathrm{SWF}$. In the LU-group about $11 \%$ felt $8 \mathrm{~V} \mathrm{VST}$, in the LN-group about $60 \%$ and in the $\mathrm{N}$-group $89 \%$. Testing temperature sensation was given up prematurely because the results in controls were unsatisfactory.

Both palmar and dorsal sides of the hands should be tested for sensation. The thresholds for protective sensation are $2.0 \mathrm{~g} \mathrm{SWF}$ and $8 \mathrm{~V}$ for vibration sense. It is recommended that Semmes-Weinstein monofilaments should always be used for early detection of loss of protective sensation.

\section{Introduction}

Nerve damage is a major problem in the treatment of leprosy patients and often results in an irreversible loss of sensation. The disabilities resulting from loss of sensation and trauma inevitably have a great impact on the patient's life. Leprosy patients with ulcers, or a history of ulcers, have clearly demonstrated the loss of protective sensation in that area on the hand. Areas without deformities, however, might already have lost their protective sensation without there being any visible signs of nerve damage (WHOdisability grading 1). These areas, therefore, might be at risk and need to be identified as

‡Correspondence: Kapelweg 15, 3566 MK Utrecht, The Netherlands. 

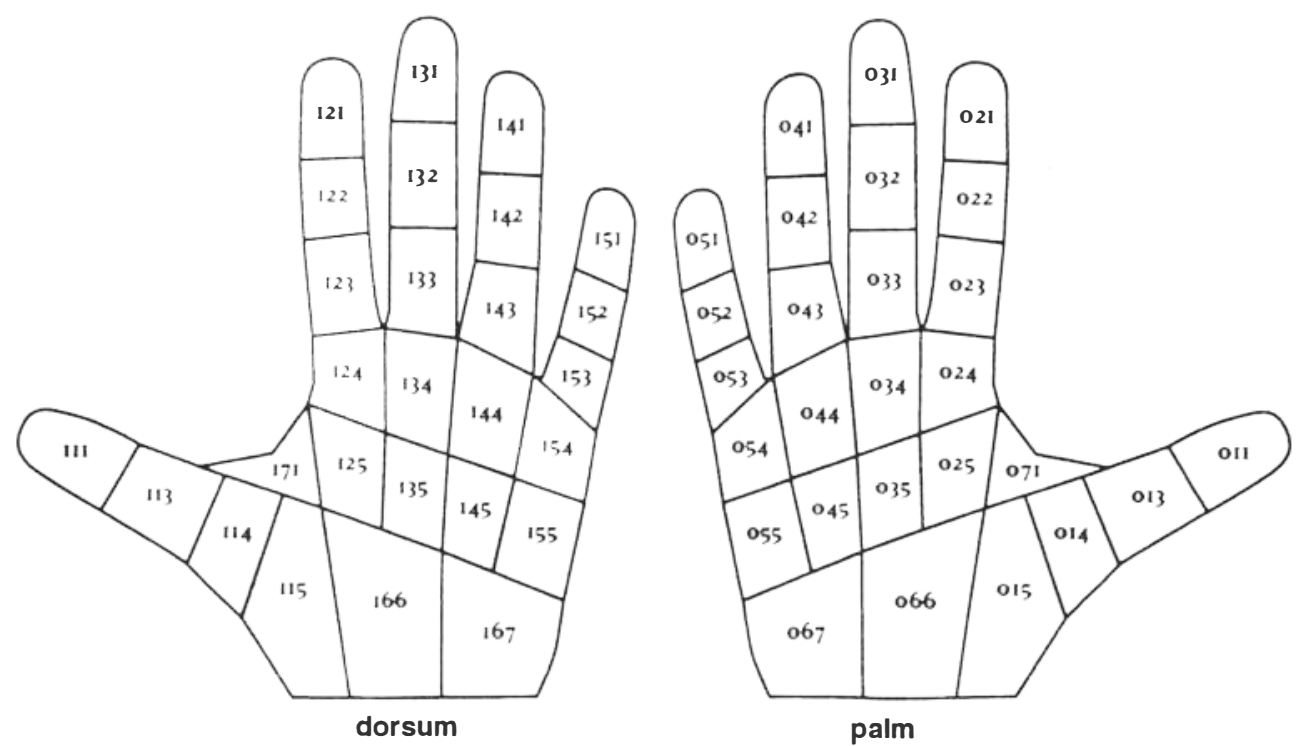

Figure 1. Chart designed according to Von Prince, ${ }^{7}$ used for mapping the hand.

soon as possible. At first sight the choice for monitoring peripheral nerve function and protective sensation thresholds would be pain sensation testing, but for reasons of reproducibility and hygiene it was decided instead to measure touch-pressure, vibration and temperature sense. Semmes-Weinstein monofilaments (SWF) are reliable tools for testing light-touch sensation, ${ }^{1}$ and have been used to detect nerve damage in the hand, ${ }^{2}$ disability grading and follow-up of nerve recovery control. ${ }^{3}$ SWF and the biothesiometer (for testing the vibration sense) have been used to measure protective sensation levels on the feet of leprosy ${ }^{4,5}$ and diabetic ${ }^{6}$ patients. Quite complicated instruments have been used in the past for measuring thermal sensibility and it was felt that the Thermo Sensation Tester could be used in estimating the thermal sensibility in the hand.

\section{Materials and method}

All the leprosy patients included in this study were registered at the McKean Rehabilitation Centre in Chiang Mai, Northern Thailand, which specializes in the treatment and rehabilitation of leprosy patients. The control subjects (nonleprosy) lived in the vicinity of the Centre or were patients attending a skin clinic in Chiang Mai. The exclusion criteria for both leprosy and control subjects were: diabetes mellitus, compression neuropathy of the upper extremity, drug or chemically induced neuropathy, and patients in reaction. Patients who could not complete the tests were also excluded. Name, age, sex, occupation and type of leprosy were recorded. Occupations were classified into three groups: heavy work, i.e. farming, labouring; mixed work, i.e. truck-driving, cleaning; and light work, i.e. teaching, office work and retired subjects. Absorptions, ulcers, cracks, blisters and scars on the hands were recorded in a drawing using a modified chart based on Von Prince's design ${ }^{7}$ (Figure 1). 
We selected 3 areas:

Areas on the dominant hand of a control subject ( $\mathrm{N}$-area $)-5$ points were tested on the palmar side: the distal area of the thumb, index and little finger and the proximal part of the thenar and hypothenar area, i.e. area $011,021,051,015$ and 067 on the chart. Controls were matched to the sex difference in leprosy $(2: 1)$.

Areas on the hands of leprosy patients with ulcers or a history of ulceration (LU-area). Tested points were on the perimeter of an ulcer, or other lesions, which were unquestionably of neuropathic origin. The number of areas tested on each patient was dependent on the number of lesions.

Areas without visible damage on the hands of leprosy patients ( $\mathrm{LN}$-area). Areas were always located distally on the digits and proximally on the palm of the hand; whenever possible, they were selected as in group 1.

A total of 150 subjects was tested, of whom 31 were controls $(\mathrm{M}: \mathrm{F}=21: 10$, mean age 36.6 years and age range $17-62$ years), and 119 leprosy patients $(\mathrm{M}: \mathrm{F}=82: 38$, mean age $52 \cdot 2$ and age range $12-93$ years).

All tests were carried out in a quiet room and all subjects were blindfolded. Each patient sat at a table with his/her hand resting comfortably on a pillow. Following an adequate explanation and demonstration of the methods of testing, the selected areas were tested. A set of $5 \mathrm{SWF}$ was used: $0 \cdot 5,2 \cdot 0,5 \cdot 5,11 \cdot 0$ and $29 \cdot 0 \mathrm{~g}$ (index number 3.61, $4 \cdot 31,4 \cdot 74,5 \cdot 07$ and $5 \cdot 46$, respectively) according to the technique described by Semmes $\&$ Weinstein. ${ }^{8}$ Each SWF was applied 3 times to the respective area; 2 or 3 correct answers out of 3 tests was recorded as a positive score, and 1 or no correct answer as a negative score. The monofilaments were used randomly and the Pressure Sensation Threshold (PST) was determined by selecting the smallest SWF with a positive score. Care was taken that there were no movements of the tested hand when the SWF was applied in order to prevent any stimulation of the proprioceptive fibres.

The Vibratory Sensation Threshold (VST) was measured for all selected areas with the biothesiometer (model PVD, Bio-Medical Instrument Company, Newbury, Ohio, USA) with a fixed frequency of $120 \mathrm{~Hz}$ and a changing output of $0-50 \mathrm{~V}$ (the square voltage divided by 100 is the amplitude in micrometers). Subjects were tested following the technique described by Bloom et al. ${ }^{9}$ Each selected area was tested 3 times from which the VST mean was calculated.

The Thermal Sensation Threshold (TST) was tested with a Thermo Sensation Tester, developed by the WHO. ${ }^{10}$ Randomly the normal or the hot tip was placed on the respective areas. Subjects again responded verbally to the stimulus. Each selected area was tested 3 times with the hot tip; 2 or 3 correct answers was a positive score, and 1 or no correct answer a negative score.

\section{Findings and results}

The composition of the stratified areas is shown in Table 1.

\section{DISTRIBUTION OF ULCERS}

In 119 leprosy patients, 65 had a total of 151 ulcers, cracks, blisters and burn wounds. The type of lesion and the sex distribution are shown in Table 2. 
Table 1. Area comparisons of all tested leprosy patients and controls

\begin{tabular}{lccc}
\hline & $\begin{array}{c}\text { N-areas } \\
(n=165)\end{array}$ & $\begin{array}{c}\text { LN-areas } \\
(n=170)\end{array}$ & $\begin{array}{c}\text { LU-areas } \\
(n=361)\end{array}$ \\
\hline $\mathrm{M}: \mathrm{F}$ & $105: 60$ & $102: 68$ & $278: 83$ \\
Mean age (yr) & $36 \cdot 6$ & $49 \cdot 3$ & $52 \cdot 1$ \\
Age range (yr) & $17-62$ & $25-79$ & $12-92$ \\
Occupation ratio (h:m:1) & $60: 40: 65$ & $64: 33: 73$ & $127: 37: 197$ \\
& & & \\
\hline
\end{tabular}

The lesions were on the palm in $48 \%$ and on the dorsal aspect in $52 \%$. On the palm they were most frequently situated on the base of the hypothenar eminence (near the pisiform bone), on the tips of the thumb and index and on the proximal phalanx of the middle finger. On the dorsum the lesions were mostly located on the thumb, on the MCP of the index and on the midphalanx of the ring finger. The distribution of the lesions on the palm and dorsum is shown in Figure 2.

PST

The cumulative percentage of the PST for all areas of the LU-, LN- and N-groups is shown in Figure 3.

The type of occupation and PST in the N-group is shown in Table 3.

In the case of the $\mathrm{N}$-group $13 \%$ of the areas revealed a PST higher than $0.5 \mathrm{~g}$; the control subjects were able to detect the $2 \cdot 0 \mathrm{~g} \mathrm{SWF}$ in all areas.

VST

Of the areas tested in the N-group, $11 \%$ had a VST higher than $8 \mathrm{~V}$, whilst in the LUgroup $90 \%$ of the areas tested had a VST higher than $8 \mathrm{~V}$. The cumulative frequency of the VST of the LU-, LN- and N-groups is shown in Figure 4.

TST

The TST of the areas tested in the N-group are presented in Table 4.

When it became apparent that a remarkably high percentage $(39 \%)$ of the areas in

Table 2. Type and sex-difference for all current lesions

\begin{tabular}{lrrr}
\hline & Female & Male & All \\
\hline Blister & 3 & 9 & 12 \\
Burn & 0 & 11 & 11 \\
Crack & 10 & 20 & 30 \\
Ulcer & 24 & 74 & 98 \\
All & 37 & 114 & 151 \\
\hline
\end{tabular}




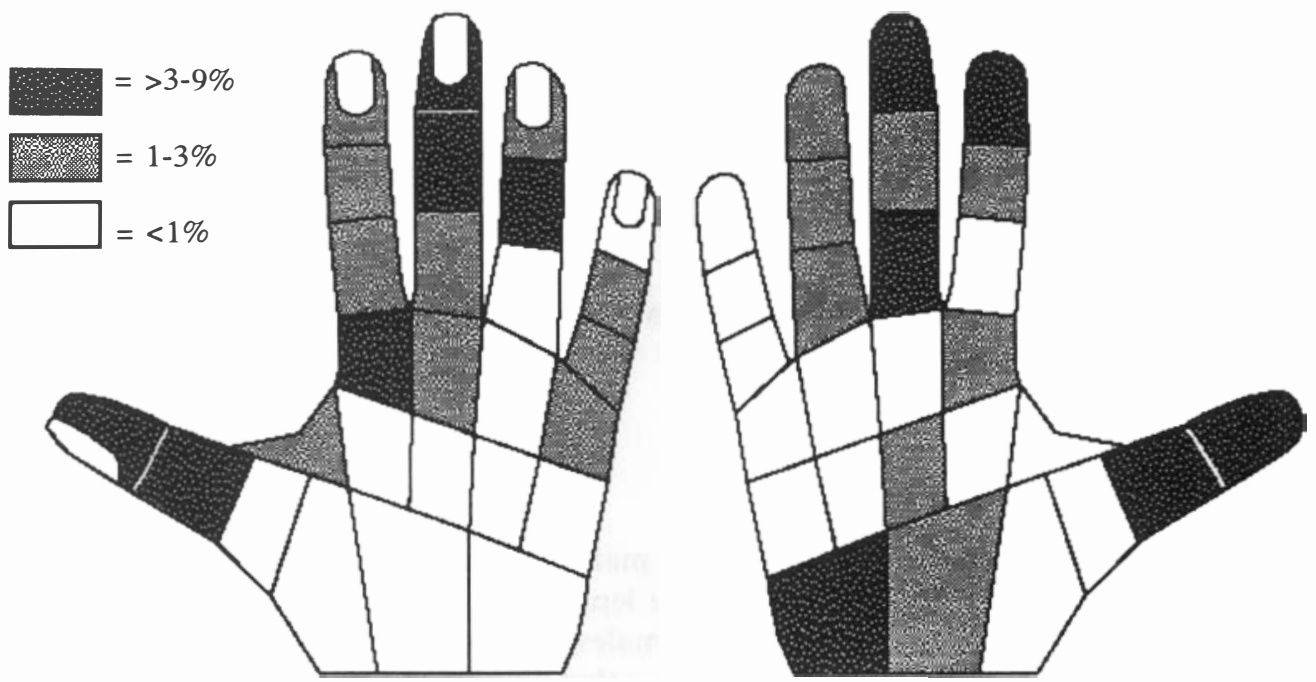

Figure 2. Distribution of lesions on the palm and dorsum of the leprosy-affected hand (expressed as percentage of all lesions).

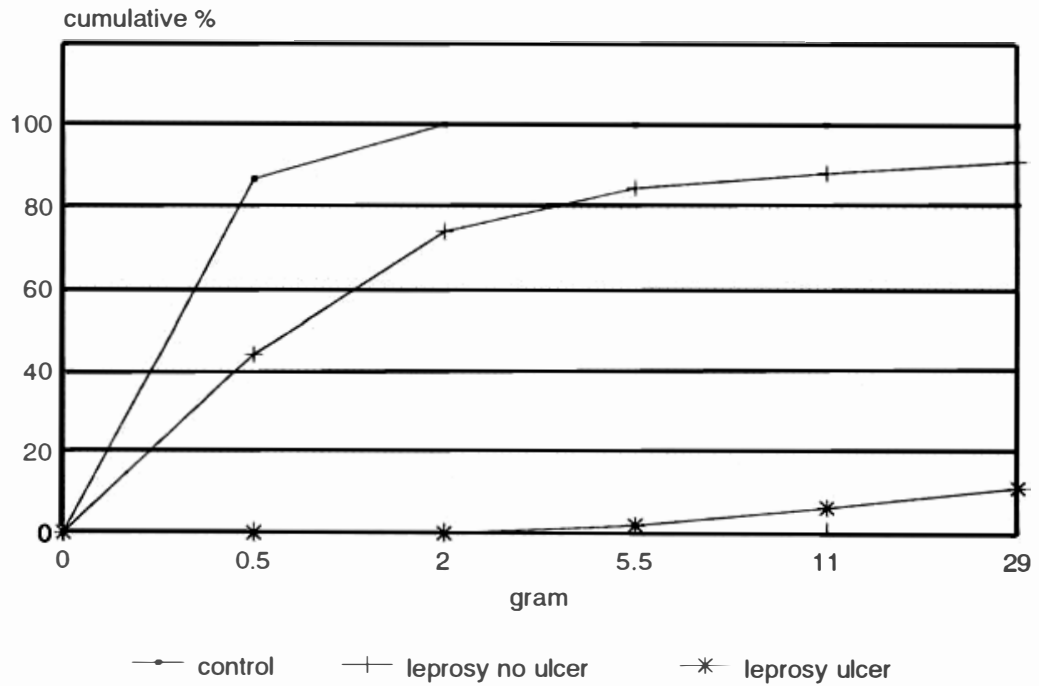

Figure 3. Cumulative percentage of pressure sensation thresholds of areas of the LU-, LN- and N-groups. 
Table 3. PST for each type of occupation in the N-group

\begin{tabular}{lccc}
\hline Weight $(\mathrm{g})$ & $\begin{array}{c}\text { Heavy } \\
\text { occupation }\end{array}$ & $\begin{array}{c}\text { Mixed } \\
\text { occupation }\end{array}$ & $\begin{array}{c}\text { Light } \\
\text { occupation }\end{array}$ \\
\hline $0 \cdot 5$ & $48(80)^{*}$ & $35(88)$ & $60(92)$ \\
$2 \cdot 0$ & $12(20)$ & $5(12)$ & $5(8)$ \\
all & $60(100)$ & $40(100)$ & $65(100)$ \\
\hline
\end{tabular}

* Numbers in parentheses are percentages.

the N-group produced no response to the hot tip of the Thermo Sensation Tester, it was decided not to proceed with this test.

\section{Discussion}

It is known that leprosy is more common in male than in female adults (sex ratio $2: 1$ to $3: 1)^{11}$ which resembles the sex ratio of the leprosy patients in this study. In children there is less difference between males and females, suggesting that sociodomestic factors are quite important. There is no clear evidence that women in Thailand report leprosy or ulceration less than men because of social reasons. The controls in this study are matched to this sex difference.

Lesions were seen in $52 \%$ of areas on the dorsum of the hand. There seems to be no difference in the type of lesions between men and women (see Table 2). In preventative education, patients should certainly be taught to be alert to changes on palmar and dorsal side of the hands.

This study confirms the relation between decreased light-touch sensation and ulcers.

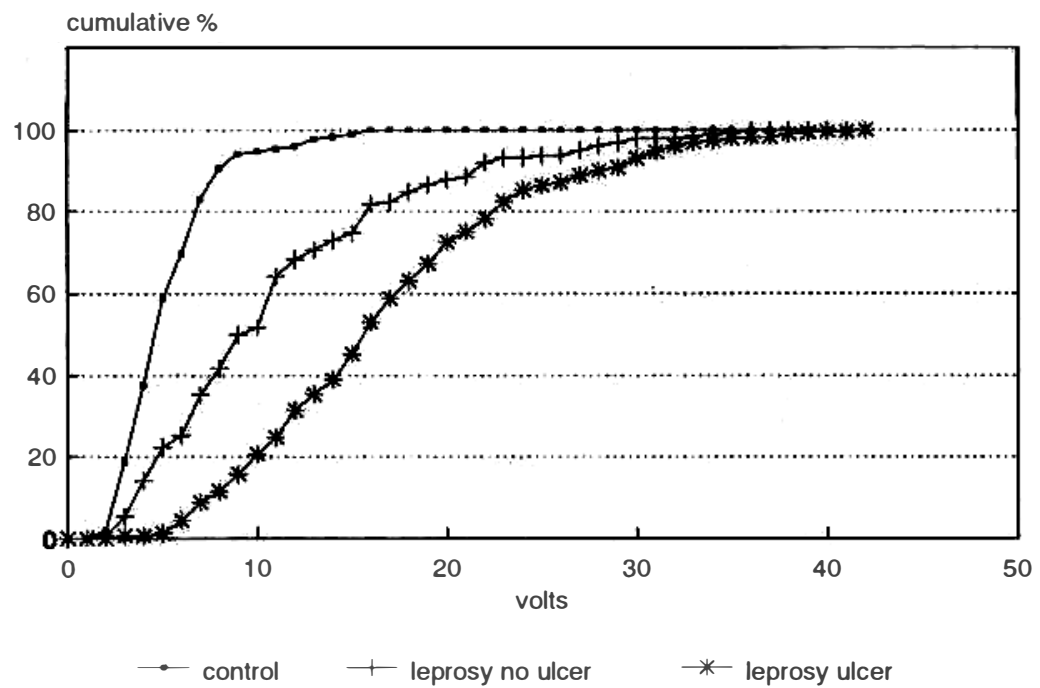

Figure 4. Cumulative percentage of vibration sensation thresholds of areas of the LU-, LN- and N-groups. 
Table 4. Sensation of the hot tip of the Thermo Sensation Tester in the N-group

\begin{tabular}{lccc}
\hline & $\begin{array}{c}\text { Temp. } \\
\text { sensation }\end{array}$ & $\begin{array}{c}\text { No temp. } \\
\text { sensation }\end{array}$ & All \\
\hline Control subject areas & $85(61)^{*}$ & $55(39)$ & $140(100)$ \\
\hline
\end{tabular}

* Numbers in parentheses are percentages.

In the $\mathrm{N}$-group, subjects were able to feel the $2 \cdot 0 \mathrm{~g} \mathrm{SWF}$ in all areas, whilst the LU-group did not respond to the $2.0 \mathrm{~g} \mathrm{SWF}$ in any area, so this cut-off point is accepted as the level of protective sensation. By applying this criterion it is possible to predict that 46 out of 170 tested areas in the LN-group are potentially at risk of developing ulcers. A follow-up study is needed to determine the predictability of this risk factor.

The present study demonstrated that SWF were easy for the examiner to use in practice and not too difficult for the subjects to understand. Questionable patients/ subject responses were easily verifiable by the use of simple techniques, i.e. taking care to apply the stimulus randomly in time and place. It is recommended that touch pressure testing be incorporated into control programmes so that areas prone to ulceration can be monitored and treated accordingly.

The WHO recommendation to use a pencil for PST measurements is open to dispute, since it is almost impossible for staff to be able to control the pressure applied, i.e. $2 \cdot 0 \mathrm{~g}$. In the $\mathrm{N}$-group, it was found that a surprising number of areas, especially on the hands of subjects with heavy occupations, were not responsive to the $0.5 \mathrm{~g} \mathrm{SWF}$ (Table 3 ). This is possibly due to the number of callouses in those areas. According to Von Prince's scale, these areas should be classified as having 'diminished protective sensation'. ${ }^{2}$ This study indicates that this scale needs to be reconsidered and possibly adjusted for use on subjects with mixed and heavy occupations.

A stimulus of $8 \mathrm{~V}$ cannot be detected in $11 \%$ of the areas tested in the $\mathrm{N}$-group and in $90 \%$ of the LU-areas. The level of protective sensation is estimated at $8 \mathrm{~V}(0 \cdot 66 \mu)$. On the basis of this criterion it could be predicted that 69 out of 170 areas in the LN-group are at risk of neuropathic ulceration. Subjects tended to have more difficulty understanding the VST test, and the degree of guess-work is more difficult to assess than the PST test. According to Williams et al.' $\mathrm{s}^{13}$ findings in VST testing, readings are inconsistent in $11 \%$ of the cases. In the light of these results and the added disadvantages of the biothesiometer (saf ety, repair, calibration, electricity dependency and costs of purchase) ${ }^{14}$ it was concluded that this apparatus is inappropriate. The use of tuning forks might be considered.

It was assumed that the Thermo Sensation Tester would be useful in TST testing, but experience has shown that in the $\mathrm{N}$-group there was a failure to distinguish the hot tip from the normal tip in a surprising $39 \%$ of the tested areas. The conclusion must be drawn, therefore, that the Thermo Sensation Tester is of little or no use in assessing hand nerve functions.

It must be acknowledged that loss of protective sensation is not the only factor of development in ulcers. Other factors include loss of autonomic (dryness) and motorfunction (clawing), the way in which hands are used and the degree of care they receive. The prevention of diability is an attempt to improve hand-care in general and to adapt 
tools to insensitive hands. These are essential elements of treatment, and they should be extended for subjects with areas at risk, and should be incorporated into the patient's general life in such a way that no new stigmas are created as a result.

\section{Conclusion}

Within the limitations of this study it is feasible to conclude that:

Distribution of lesions indicates a remarkable high percentage $(52 \%)$ on the dorsum of the hand. The registration and monitoring of this occurrence should not be overlooked in the future.

The level of protective sensation in the hand is $2.0 \mathrm{~g} \mathrm{SWF}$. A prospective study is needed to determine the predictability of developing neuropathic ulceration or other lesions.

The Von Prince standard sensory threshold scale ${ }^{7}$ needs to be adjusted.

Biothesiometry is both more complicated and less accurate than the SWF in distinguishing areas in control subjects from lesion areas in leprosy patients.

The newly-developed Thermo Sensation Tester is deemed inappropriate for measuring thermal sensibility thresholds on the hand.

\section{Acknowledgments}

The authors wish to extend special thanks to Professor F. G. I. Jennekens, Head of the Department of Neuromuscular Diseases, University of Utrecht, The Netherlands, to W. J. Brandsma, Physiotherapist and J. H. Richardus, Physician of the McKean Rehabilitation Centre, for their encouragement and critical comments, to patients and staff of the McKean Rehabilitation Centre for their co-operation, and to Kees Schreuders for his help in producing the graphs and illustrations.

\section{References}

${ }^{1}$ Bell-Krotoski J, Tomancik E. The repeatability of testing with Semmes-Weinstein monofilaments. $J$ Hand Surg, 1987; 12-A: 155-61.

2 Palande DD, Bowden REM. Early detection of damage to nerves in leprosy. Lepr Rev, 1992; 63: 60-72.

3 Naafs B, Dagne T. Sensory testing: a sensitive method in the follow-up of nerve involvement. Int J Lepr, 1977; 45: 364-8.

${ }_{5}^{4}$ Birke JA, Sims DS. Plantar sensory threshold in the ulcerative foot. Lepr Rev, 1986; 57: 261-7.

${ }^{5}$ Hammond CJ, Klenerman P. Protective sensation in the foot in leprosy. Lepr Rev, 1988; 59: 347-54.

${ }^{6}$ Holewski JJ et al. Aesthesiometry: Quantification of cutaneous pressure sensation in diabetic peripheral neuropathy. J Rehab Res, 1988; 25: 1-10.

7 Von Prince K, Butler B. Measuring sensory function of the hand in peripheral nerve injuries. Am J Occ Ther, 1967; 21: 385-95.

8 Semmes J, Weinstein S. et al. Somatosensory changes after penetrating brain wounds in man. Cambridge, 1960, Harvard University Press, pp. 4-11.

9 Bloom S et al. Use of a biothesiometer to measure individual vibration thresholds and their variation in 519 non-diabetic subjects. Br Med J, 1984; 288: 1793-5.

10 Srinivasan H, Stumpe B. Value of thermal sensibility testing in leprosy diagnosis in the field-field trial of a pocket device. Lepr Rev, 1989; 60: 317-26.

11 Hunter's Tropical Medicine, ed. G. T. Strickland, W. B. Saunders Company, Philadelphia, 7th edition, p. 485 . 
12 Von Prince K, Butler B. Measuring sensory function of the hand in perif eral nerve injuries. Am J Occ Ther, 1967; 21: 385-95.

${ }^{13}$ Williams G. et al. Variability in vibration perception threshold among sites: a potential source of error in biothesiometry. Brit Med J, 1988; 269: 233-5.

14 Watts GF, Shaw KM, Long G. Warning on the use of the biothesiometer. Daib Med, 1986; 3: 91. 